\author{
Burghardt Klöden $^{\mathrm{a}, \mathrm{c}}$, Erik Rybacki ${ }^{\mathrm{b}}$, Carl-Georg Oertel ${ }^{\mathrm{a}}$, Werner Skrotzki ${ }^{\mathrm{a}}$ \\ ${ }^{a}$ Technische Universität Dresden, Institut für Strukturphysik, Dresden, Germany \\ ${ }^{\mathrm{b}}$ Geoforschungszentrum Potsdam, Potsdam, Germany \\ ${ }^{c}$ Fraunhofer Institut für Fertigungstechnik und Angewandte Materialforschung, Dresden, Germany
}

\title{
Grain refinement and texture formation in torsion deformed NiAl
}

Torsion at elevated temperatures and pressures was used to severely deform the intermetallic compound NiAl. The microstructure and texture as a function of shear strain were investigated by orientation imaging microscopy and diffraction of synchrotron radiation, respectively. The results show that at high shear strains a steady state grain structure and texture develops by continuous dynamic recrystallisation. The lowest grain size achieved is in the micron range, the main texture component is $\{110\}<100>$. It is concluded that high-strain torsion may open new possibilities in terms of grain refinement, texture formation and ductilisation of NiAl.

Keywords: NiAl; Torsion; Microstructure; Texture; Ductility

\section{Introduction}

$\mathrm{NiAl}$ is an intermetallic compound which due its high melting temperature, low density, high corrosion resistance and moderate creep resistance has a high potential for high-temperature structural applications. However, because of the low-temperature brittleness of polycrystalline NiAl, technical applications are still lacking. Thus, similar to other in-

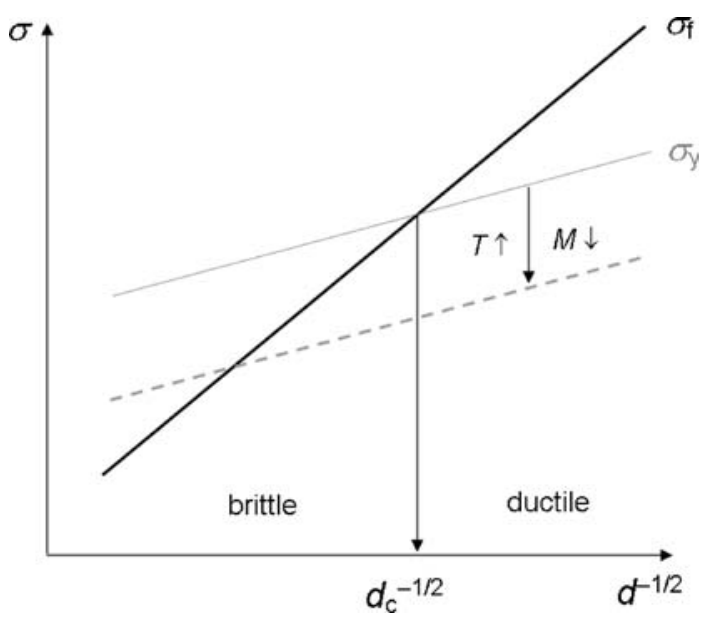

Fig. 1. Grain size $d$ dependence of the fracture stress $\sigma_{\mathrm{f}}$ and the yield stress $\sigma_{\mathrm{y}}$ defining the brittle-to-ductile transition grain size $d_{\mathrm{c}}$. Dashed line shows the effect of texture and temperture on $\sigma_{\mathrm{y}}$ in the case of decreasing Taylor factor $M$ and increasing temperature yielding an increase in $d_{\mathrm{c}}$. termetallics, it is a great challenge to ductilise NiAl. Attempts to reduce the resistance for secondary slip by alloying in order to fulfill the von Mises criterion have not been successful [1]. Another way to approach this problem is by changing the grain structure and texture on purpose. As the yield stress increases much more slowly with decreasing grain size than the fracture stress [2], grain refinement should help to reach the ductile field (Fig. 1). This field can be extended by changing the texture in such a way that the Taylor factor is reduced or by increasing the temperature. Thus, in order to optimise the desired mechanical properties by thermomechanical treatment it is of particular interest to understand the mechanism of grain refinement and texture formation during deformation. Therefore, it was the aim of the present work to use severe plastic deformation (SPD) by torsion, a new processing route effective in grain refinement [3]. Torsion is a deformation mode allowing the study of microstructure and texture with strain. To do this, methods with high spatial resolution had to be used which are given by electron back-scatter diffraction (EBSD) in the scanning electron microscope (SEM) and by texture measurements with high-energy synchrotron radiation. Preliminary results have already been reported elsewhere $[4,5]$.

\section{Experimental}

The polycrystalline NiAl samples investigated had a nearly stoichiometric composition (Ni-50.1 Al). Depending on the thermomechanical history of the material, different initial preferred orientations $(<100\rangle,<110\rangle$ and $<111>$ ) were parallel to the torsion axis. The microstructure consisted of equiaxed grains with an average grain size of about $50 \mu \mathrm{m}$. Torsion samples were cylinders with length and diameter of $10 \mathrm{~mm}$.

Deformation through torsion was done with a Paterson rock deformation machine [6] at the Geo Research Center Potsdam. The confining pressure produced by argon gas creates large contact forces between the parts of the sample assembly as well as between the external load cell and the upper end of the sample assembly. Due to this, a torque can be transmitted to the sample by friction through the movement of the torsion actuator. The torque is limited by possible slipping between the components of the sample column. The shear stress-shear strain curves were calculated from the measured torque and twist data according to the procedure given in $[6,7]$. The measured torque was cor- 


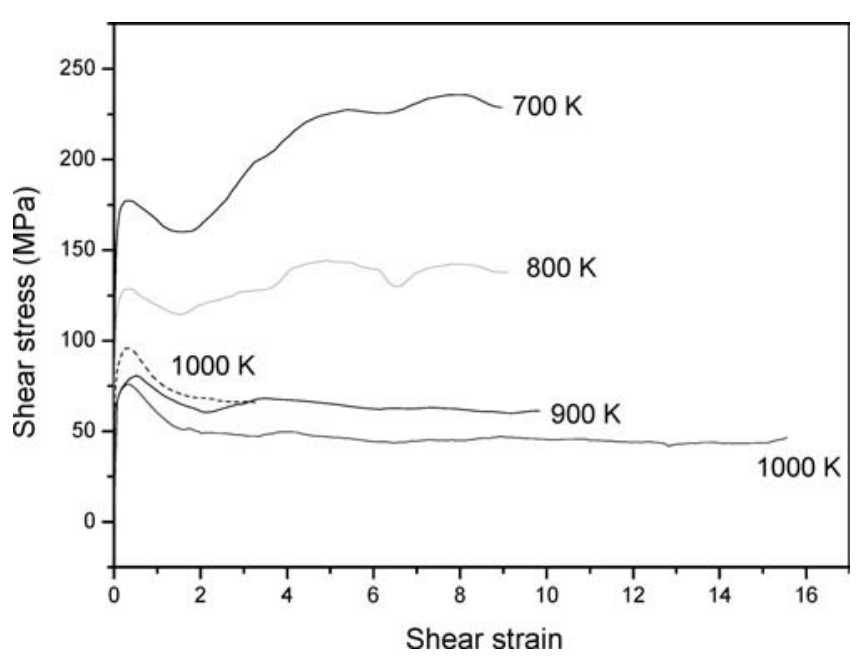

Fig. 2. Shear stress - shear strain curves of $<111>$ oriented samples at different temperatures. The dashed line refers to a higher shear deformation rate of $2.2 \times 10^{-4} \mathrm{~s}^{-1}$.

rected for apparatus compliance and steel jacket strength (accuracy $\approx 3 \%$ ). Due to sample shortening during torsion the shear strain rate slightly increased with shear strain (at maximum factor 1.4). A report on this effect, known as the Swift effect [8], will be published separately. The following parameters were used: confining pressure: $0.4 \mathrm{GPa}$, temperatures: $700-1000 \mathrm{~K}$, maximum shear strain: 19 , maximum shear strain rate: $3.6 \times 10^{-5} \mathrm{~s}^{-1}$ (exception $2.2 \times$ $10^{-4} \mathrm{~s}^{-1}$, see Fig. 2).

To investigate the microstructure, spatial distribution of orientation and misorientation, maps were constructed from EBSD scans made with an SEM (LEO 1530) on tangential axial sections cut at different distances from the torsion axis in the radial direction, i. e. at different shear strains. The orientation analysis was done with the software Channel 5 from HKL, Hobro, Denmark. The grain structure was characterised by grain boundary misorientations $\geq 3^{\circ}$. The grain shape was approximated by an ellipse with the same area as the grain. Thus, the aspect ratio $a / c$ (length of long $a$ to short half axis $c$ ) and the inclination angle of the grain long axis with respect to the shear direction could be measured. The grain size $d$ was taken as the diameter of a circle with the same area as the ellipse.

To get the starting texture, global texture measurements on the entire coarse-grained torsion cylinders were performed by neutron diffraction [9] at the GKSS Research Centre, Geesthacht. The local texture of the fine-grained torsion deformed samples was measured with high-energy synchrotron radiation [5] at DESY-HASYLAB, Hamburg. The synchrotron radiation opens up the possibility of investigating representative small sample volumes $1 \mathrm{~mm} \times$ $1 \mathrm{~mm} \times(0.7-1) \mathrm{mm}$ along the radial direction of the torsion samples. The textures are presented in sections of the orientation distribution function (ODF) calculated from the measured pole figures ((100), (110), (111)) using the program developed by Dahms and Eschner [10] based on the iterative series expansion method. The Euler angles are used in the Bunge notation [11]. To account best for the monoclinic symmetry of simple shear, the crystal and sample reference systems were defined as [100]|lshear direction, [010]|lshear plane normal, [001] IItransverse direction [12].

\section{Results}

\subsection{Shear stress - shear strain curves}

The torsion behaviour of the NiAl polycrystals is shown exemplarily by the shear stress - shear strain curves for $<111>$ oriented samples in Fig. 2. The curves are characterised by a peak at low strains and high temperatures, which is followed by softening and a steady state at high strains (Fig. 2). The position and height of the peak depends on the initial orientation. It evolves at low shear strain values of $\gamma=0.1-0.2$ for initial $<110>$ and $<111>$ orientation and at an order of magnitude higher values of $\gamma=1-2$ for initial $<100>$ orientation. The peaks are highest for $<100>$ samples. At temperatures below $900 \mathrm{~K}$, for $<110>$ and $<111>$ samples the shear stress-shear strain curves exhibit from $\gamma=2$ onwards a parabolic hardening to a steady state stress. This effect, which is more pronounced for the $<111>$ samples may be attributed to the relative increase in the harder cube component (see below). Increasing the shear strain rate in general leads to higher shear stresses.

An activation analysis of the creep process done by strain rate and temperature jumps yields a power-law stress exponent increasing with decreasing temperature from about 6 to 14 and an activation energy of about $3 \mathrm{eV}$ slightly decreasing with increasing stress [13]. This behaviour can be explained by a creep mechanism controlled by cross slip of screw dislocations.

\subsection{Microstructure}

Figure 3 a shows that (i) after shear strains $>2$ the overall grain size is already much smaller than the initial one of about $50 \mu \mathrm{m}$ and (ii) grain refinement increases with decreasing temperature for constant initial orientation. Within the shear strain range investigated, a steady state grain size is reached for all samples. The initial orientation does not affect the steady state grain size, which is in the range of $\approx 3 \mu \mathrm{m}$ at $800 \mathrm{~K}, \approx 7 \mu \mathrm{m}$ at $900 \mathrm{~K}$ and $\approx 10 \mu \mathrm{m}$ at $1000 \mathrm{~K}$. Separation of low and high angle grain boundaries (LAGBs and HAGBs, misorientations $<$ and $>15^{\circ}$, respectively) (Fig. 3b) shows that for the highest strains reached the grain size determined from HAGBs is about a factor $1.2(700 \mathrm{~K})$ or $1.3(800 \mathrm{~K})$ larger than that determined from all grain boundaries representing the subgrain size.

The initial fraction of LAGBs is about $15 \%$. With increasing shear strain the LAGB fraction goes over a maximum at a shear strain smaller than about 2 and then decreases to a steady state value (Fig. 4). The maximum value is about $70 \%$, the steady state fraction about $20-$ $40 \%$, i.e. the microstructure is dominated by HAGBs at high shear strain. In addition, there is a general trend for the steady state value to increase with temperature in the sequence $\langle 110\rangle,<111\rangle,<100\rangle$. This may be attributed to strengthening of the texture with temperature (see below).

Due to shear the initially equiaxed grains are elongated with the grain long axis being inclined to the shear direction. Figure 5 shows the shear strain dependence of the arithmetic mean inclination angle $\varphi$. There is a general tendency for the experimental values to be larger than expected from the theoretical curve for "pure" simple shear 


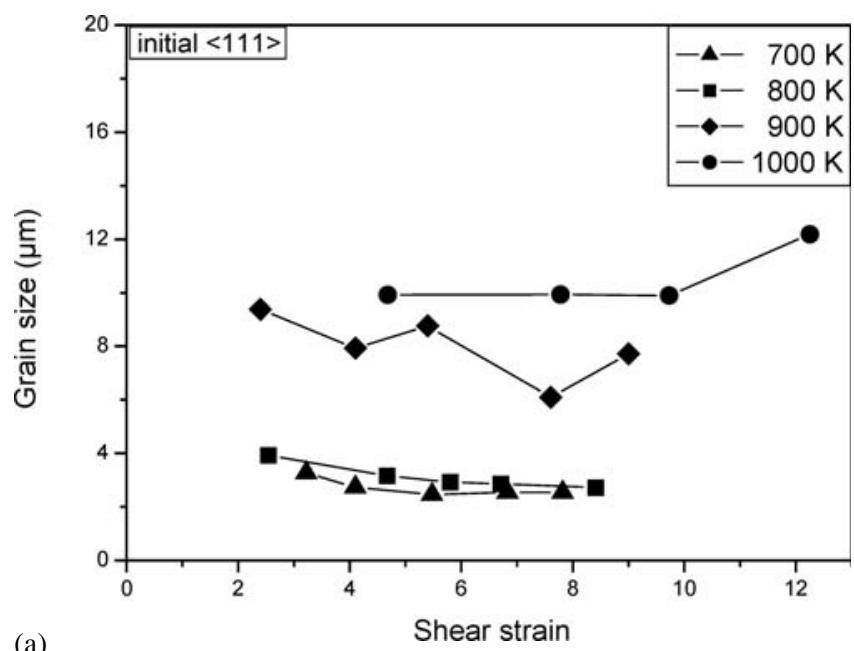

(a)

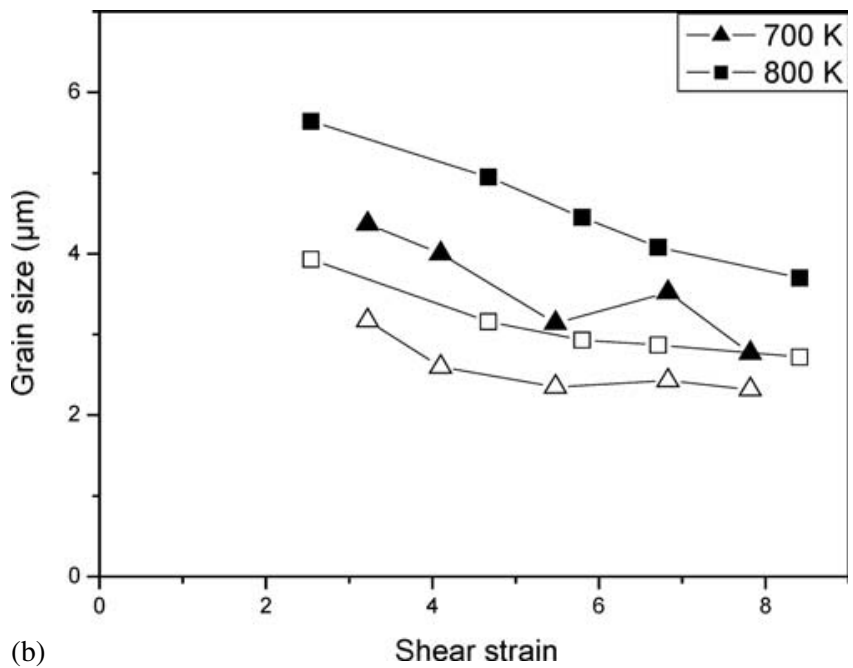

(b)

Fig. 3. Grain size subject to the shear strain of $<111>$ oriented samples at different temperatures: (a) all grain boundaries, (b) separation of grain boundaries with misorientations smaller than $15^{\circ}$ (low angle grain boundaries or subgrain boundaries, open symbols) and larger than $15^{\circ}$ (high angle grain boundaries, solid symbols).

deformation [14]:

$\Phi=1 / 2 \arctan (2 / \gamma)$

For all samples, an average value of about $30^{\circ}(T=800 \mathrm{~K}$ and $900 \mathrm{~K})$ and $20^{\circ}(T=1000 \mathrm{~K})$, is established at shear strains greater than 8 , irrespective of initial orientation. At low strains $(<100>$ : $<5,<110>$ and $<111>$ : $<3)$ and temperatures $>800 \mathrm{~K}$ the inclinations are lower and approach the curve for "pure" simple shear deformation. The average aspect ratio is constant at about 2.5-2.7 for $\gamma>3$ independent of initial orientation and temperature. For reasons of clarity the inverse aspect ratio is shown in Fig. 6. The comparison of the average aspect ratios with the theoretical values according to "pure" simple shear deformation [14]

$a / c=1 / 2\left(\gamma^{2}+2+\gamma\left(\left(\gamma^{2}+4\right)^{1 / 2}\right)\right.$

shows a pronounced deviation.

\subsection{Texture}

During torsion there is a change from the initial texture (almost cube, $<110>$ fibre, $<111>$ fibre) to a typical shear tex-

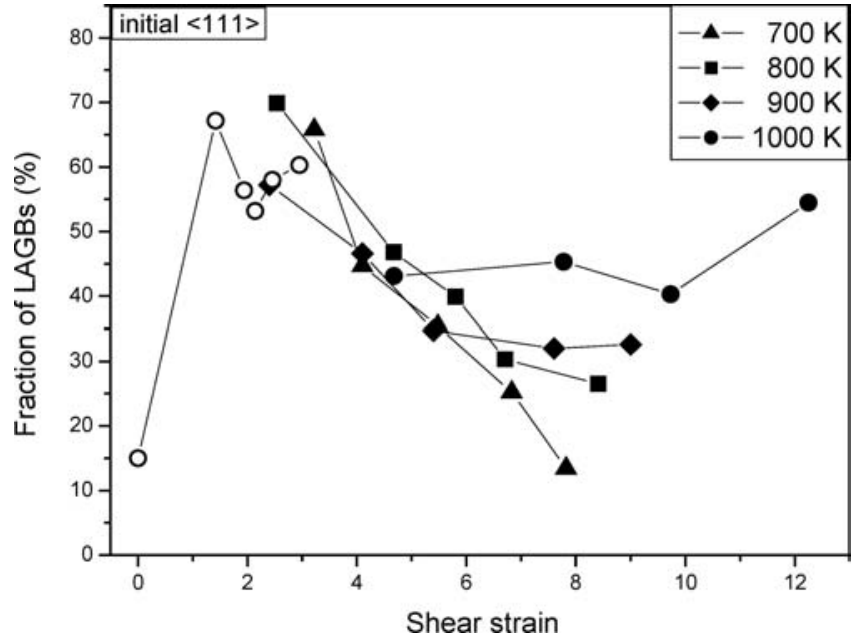

Fig. 4. Fraction of low angle grain boundaries (LAGBs) subject to shear strain of $<111>$ oriented samples deformed at different temperatures. The open symbols refer to a higher shear deformation rate of $2.2 \times 10^{-4} \mathrm{~s}^{-1}$

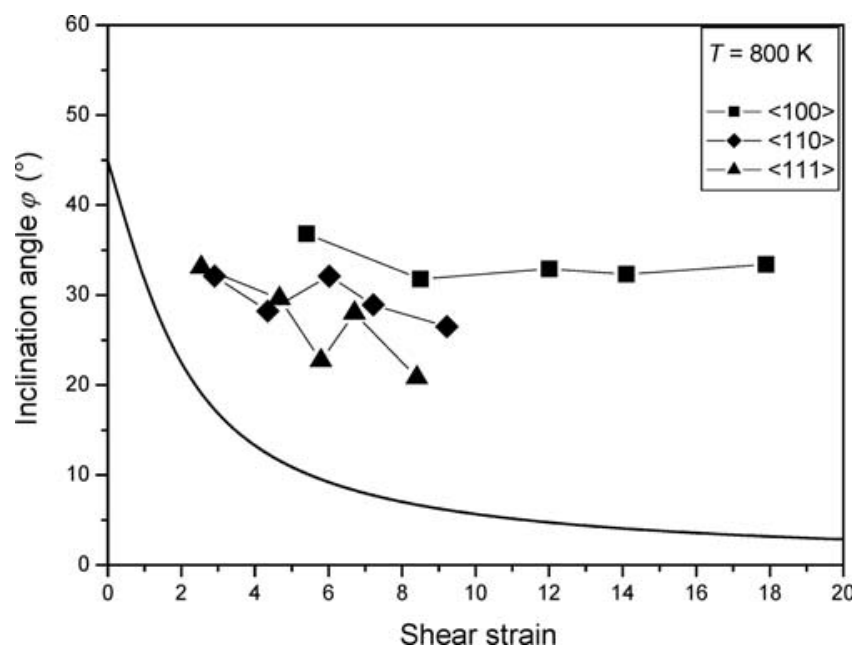

Fig. 5. Arithmetic mean inclination angle subject to shear strain for three different initial orientations at $800 \mathrm{~K}$. The solid line refers to "pure deformation".

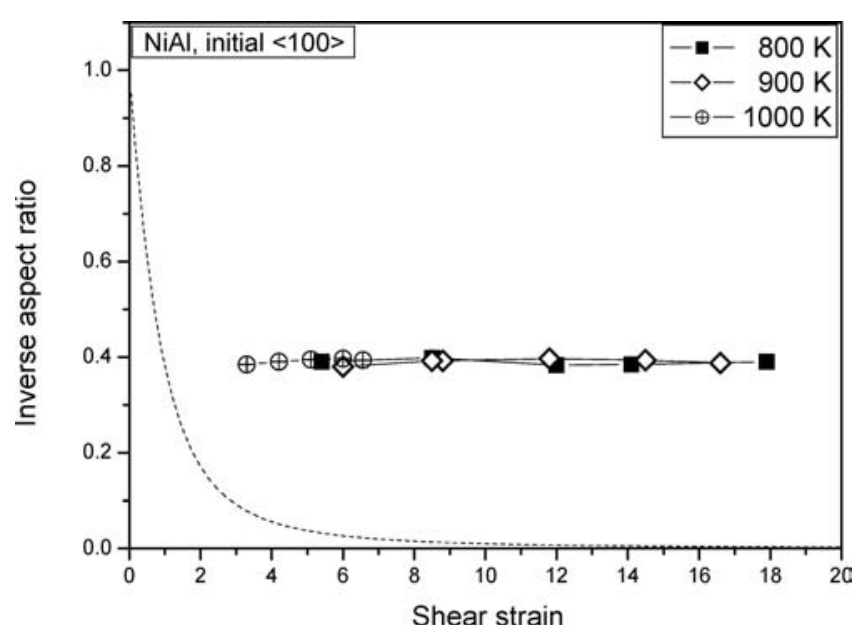

Fig. 6. Inverse grain aspect ratio subject to the shear strain for three different initial orientations at $800 \mathrm{~K}$. The solid line refers to "pure deformation".

Int. J. Mat. Res. (formerly Z. Metallkd.) 98 (2007) 4 
ture. The shear texture is characterised by two components, $\{100\}<100>$ (cube, $C$ ) and $\{110\}<100>$ (Goss, G) (Fig. 7) increasing with strain to an almost constant strength. The intensity of $G$ increases with temperature, while that of $C$ decreases independent of the initial orientation (Fig. 8). Both components deviate from the ideal position in positive $\varphi_{1}$ direction (around the transverse direction). The deviation is larger for grains containing the $C$ component and decreases with temperature (Fig. 9). In comparison, grains containing the $G$ component have a smaller deviation decreasing with strain and temperature.
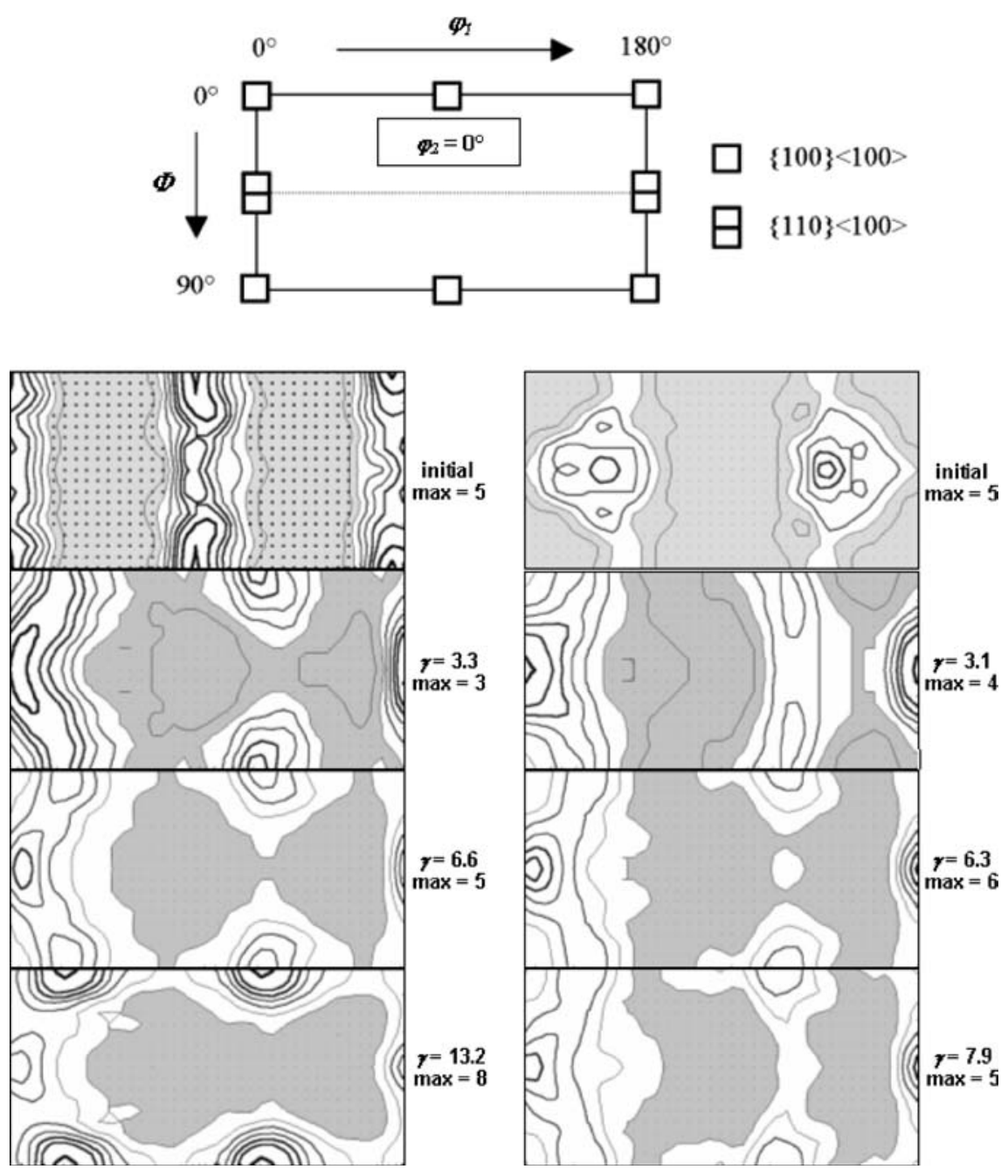

Fig. 7. ODF sections at $\varphi_{2}=0^{\circ}$ for $<100>$ (left column) and $<111>$ sample (right column) at $800 \mathrm{~K}$. Maxima are given in multiples of a random distribution, mrd. Key figure at the top shows the position of the two ideal components.
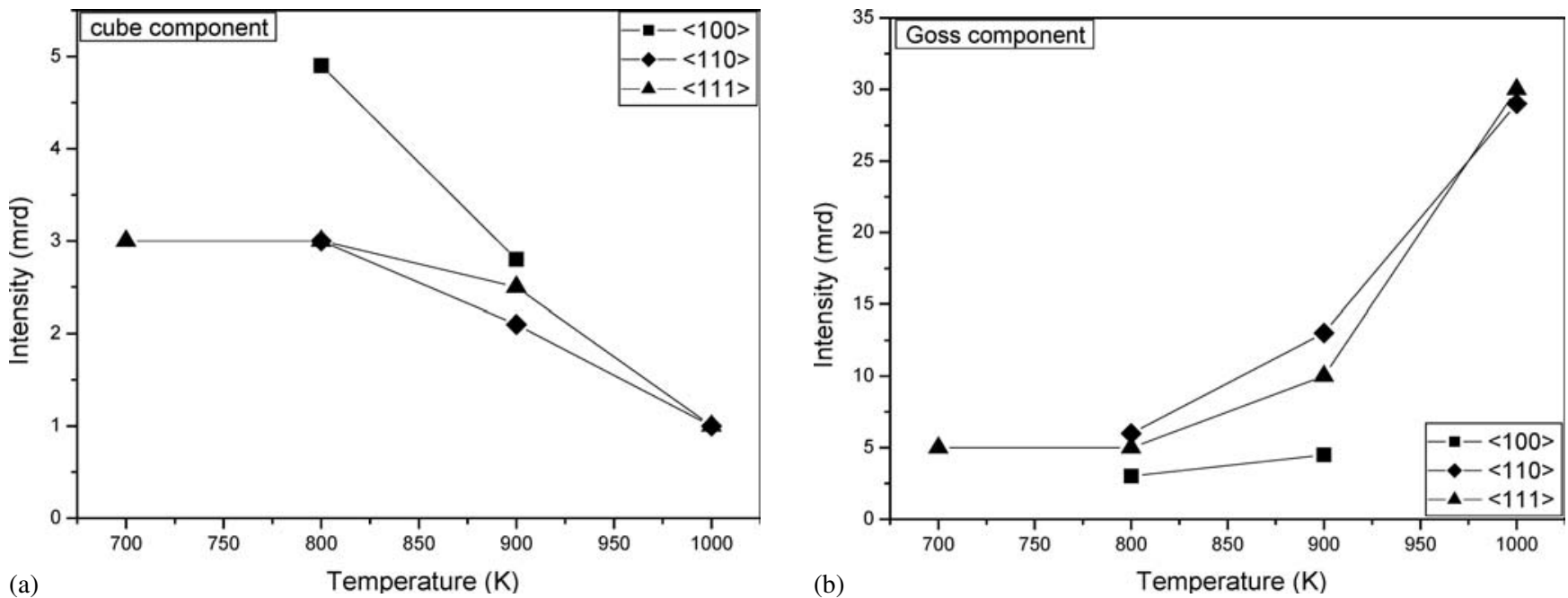

Fig. 8. Texture intensity for the cube and Goss component at constant shear strain $(\gamma \approx 8)$ for three different initial orientations.

Int. J. Mat. Res. (formerly Z. Metallkd.) 98 (2007) 4 
B. Klöden et al.: Grain refinement and texture formation in torsion deformed NiAl
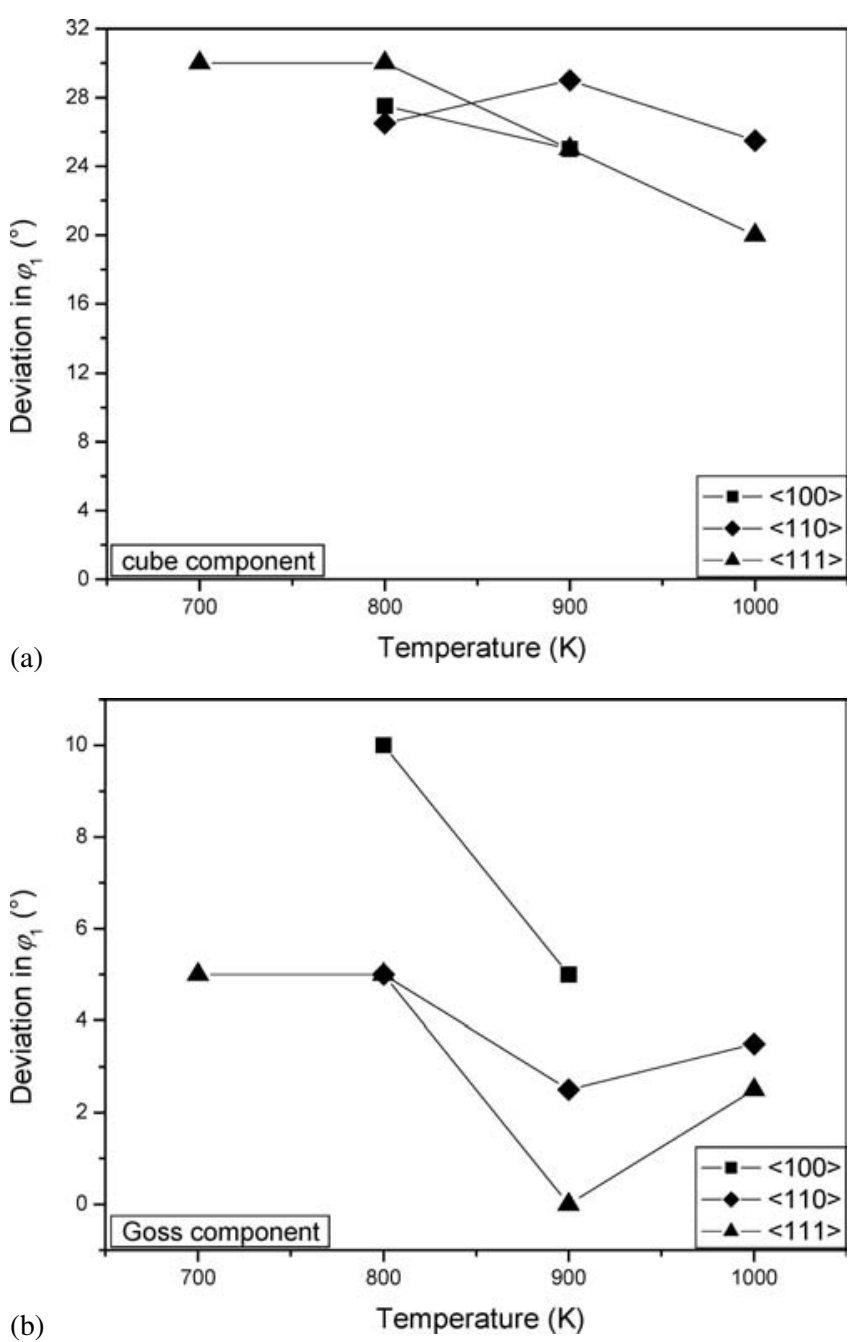

Fig. 9. Angular deviation in $\varphi_{1}$ of the cube and Goss component at constant shear strain $(\gamma \approx 8)$ for three different initial orientations.

\section{Discussion}

\subsection{Mechanism of recrystallisation}

The results have shown that $\mathrm{NiAl}$ during torsion at elevated temperatures develops the following characteristics with regard to work-hardening/softening and microstructure and texture development:

1. The shear stress - shear strain curves exhibit a single and smooth maximum followed by a slow but significant softening (or hardening) stage ending in a steady state at high strains.

2. The microstructural parameters, such as grain size, aspect ratio, grain shape foliation and fraction of high angle grain boundaries, develop in a characteristic manner with shear strain reaching a steady state. There is grain refinement up to a factor of 20.

3. A strong texture develops at high strains almost reaching saturation.

These features are characteristic of continuous dynamic recrystallisation (CDRX) [15-18]. According to this recrystallisation mechanism dislocations produced by strain hardening progressively accumulate in LAGBs (or subgrain boundaries) leading to the increase of their misorientation angle and the formation of HAGBs when a critical value of misorientation is reached. In the present case the maximum fraction of LAGBs is reached for $\gamma \approx 2$ (Fig. 4). However, not only does transformation of LAGBs to HAGBs take place, but so too does limited migration of HAGBs, mainly driven by the difference between dislocation densities of the grains located on the two sides of the boundary. On the one hand, besides usual dynamic recovery this grain boundary migration leads to the absorption of dislocations from the grain interiors, i.e. to additional softening. On the other hand it gives rise to new grain subdivision by LAGBs, which during further straining may develop to HAGBs. Hence, this continuous process of coarsening, subdivision and shearing yields a steady state microstructure of constant average grain size, aspect ratio, grain shape foliation and fraction of HAGBs. If grain boundary migration is high, i.e. the time a grain boundary moves a distance of the order of grain radius is short compared to the transformation of LAGBs to HAGBs, then this mechanism is called discontinuous dynamic recrystallisation (DDRX). DDRX is favoured by purity of the material and/or high temperature and/or low strain rate. In $\mathrm{NiAl}$ a transition from CDRX to DDRX takes place above about $1100 \mathrm{~K}$ [19]. Generally, CDRX leads to shear stress - shear strain curves as decribed in (1), above while DDRX yields oscillating curves due to the repeated production of dislocation-free grains [16]. However, additionally (i) grain boundary migration (ii) textural changes (geometrical softening) and (iii) adiabatic shear heating may also cause strain softening. So far, process (ii) has been neglected in modelling CDRX $[17,18]$.

As grain boundary migration in CDRX is limited (grain volume change of 1.7 (700 K) and 2.4 (800 K), Fig 3b), the textures observed should be "deformation textures". However, with increasing temperature the constraints of strain compatibility should be relaxed due to absorption of dislocations in the grain boundary vicinity. This leads to a close alignment of the primary slip system $\{110\}<100>$ (may also be $\{100\}<100>$ ) with the shear system $(G$ and $C$ texture components). The results have shown that the intensity and alignment of the two components are orientation and temperature dependent. Simulations of the texture development are under way.

\subsection{Prospect of ductilisation by grain refinement}

In this chapter the problem of ductilisation of $\mathrm{NiAl}$ will be discussed. Based on an extended study of the plasticity of ionic crystals with NaCl-structure, Skrotzki and Haasen [20] concluded that a necessary requirement for polycrystalline ductility is given by (i) the fulfillment of the von Mises criterion [21], i.e. the easy activation of five independent slip systems. However, to be a sufficient condition, (ii) cross slip of all types of screw dislocations must also be possible at the onset of plastic deformation in order to prevent piling-up of dislocations at obstacles such as grain boundaries. If only condition (i) is fulfilled, then (iii) grain refinement may help to decrease the brittle-to-ductile transition temperature (BDTT). This is because with decreasing grain size for a given applied stress the number of piled-up dislocations and, thus, the effective stress at the front of the pile-up necessary for crack initiation and/or propagation decreases.

How are conditions (i) and (ii) fulfilled for $\mathrm{NiAl}$ ? 


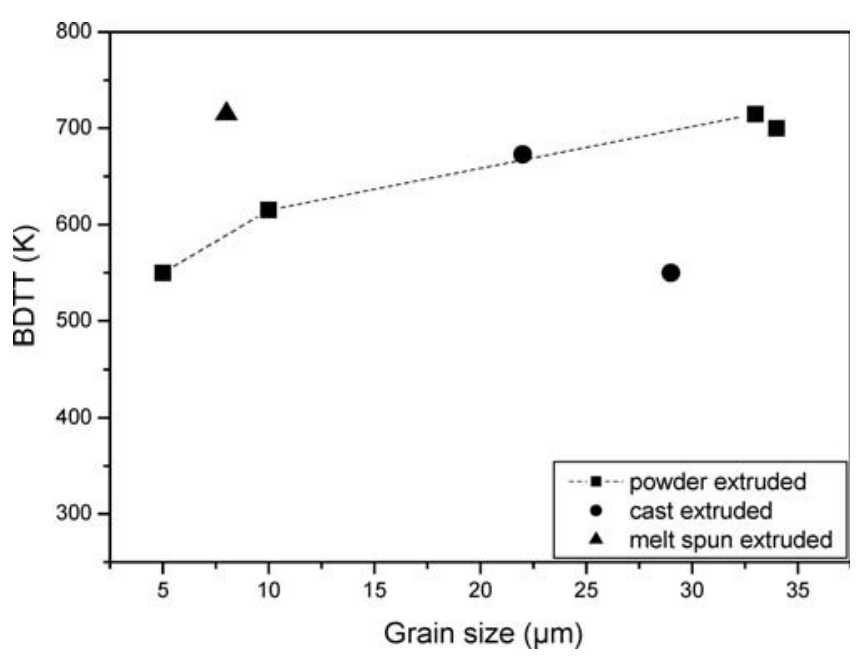

Fig. 10. Brittle-to-ductile transition temperature (BDTT) as a function of grain size for NiAl (Compilation taken from [26]). The BDTT is defined as the temperature at which for a given grain size the tensile strain to fracture is at least $5 \%$.

Condition (i): Deformation of NiAl single crystals of different orientations have shown that $\{110\}<100>$ is the primary slip system at all temperatures, while secondary slip is by $\{110\}<111>$ and $\{110\}<110>$ below and above a critical temperature $T_{\mathrm{C}}$, respectively [1]. The critical resolved shear stress for $\{110\}<110>$ drastically decreases above $T_{\mathrm{C}}$. For stoichiometric NiAl $T_{\mathrm{C}}$ is about $600 \mathrm{~K}$, it increases with deviation from stoichiometry, alloying and increasing strain rate. To fulfill the von Mises criterion, besides $\{110\}<100>$ ( 3 independent slip systems), $\{110\}<111>$ (5) or $\{110\}<110>(2)$ have to be activated. This becomes easy above $T_{\mathrm{C}}$.

Condition (ii) is fulfilled for $\langle 100\rangle$ and $<111>$ dislocations because of a compact core [22, 23]. Therefore, cross slip is a commonly observed phenomenon [24]. In contrast, $<110>$ dislocations have been reported to have a complicated core structure $[12,25]$ rendering cross slip more difficult.

Condition (iii): Grain refinement reduces the BDTT of $\mathrm{NiAl}$. This has been shown for powder-extruded NiAl samples of the same stoichiometry, purity and texture (Fig. 10) [26]. However, the situation is unclear for melt-spun- and cast-extruded samples of different origin. The lowest BDTT achieved so far is $550 \mathrm{~K}$, which, unfortunately, is about $T_{\mathrm{C}}$ and not significantly below. Therefore, if condition (ii) in general is a necessary requirement, then $T_{\mathrm{C}}$ is the inherent BDTT and all efforts should be addressed to lower this temperature. However, above $T_{\mathrm{C}}$ conditions (ii) or (iii) may limit ductility.

Different effects may weaken condition (i):

a) Change in the deformation mechanism from dislocation slip-controlled to grain boundary sliding-controlled. According to the activation analysis applied (see Section 2.1) creep in the NiAl polycrystals used is controlled by cross-slip of screw dislocations. Therefore, for grain boundary sliding to become effective at low temperatures the grain size has to be reduced further. Figure 11 gives an impression of grain refinement during SPD. If CDRX continues to lower temperatures, then for flow stresses of about $1400 \mathrm{MPa}$ [27] measured

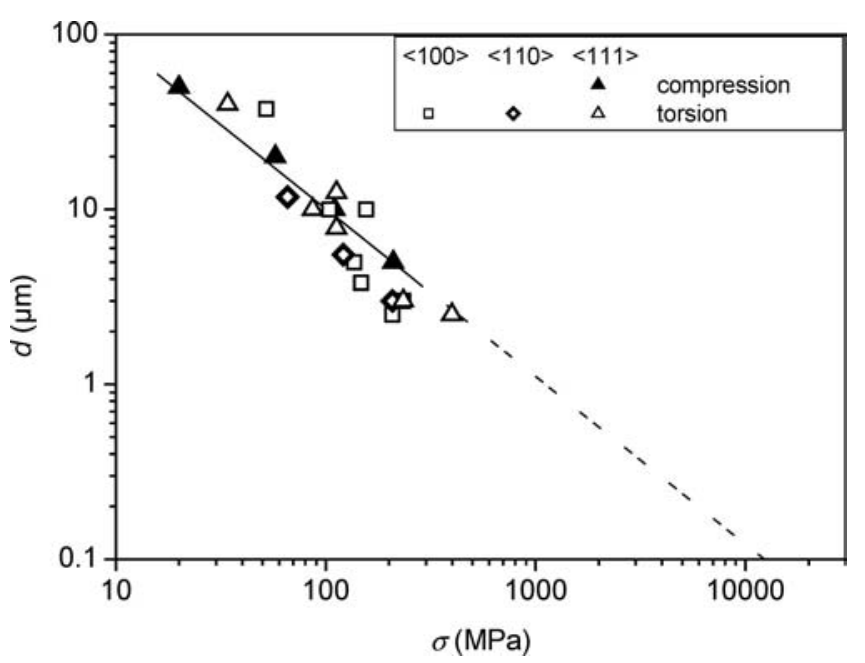

Fig. 11. Grain size as a function of axial stress (equivalent stress). The transformation of shear stress $\tau$ into equivalent stress $\sigma$ has been made according to $\sigma=3^{(1+n) / 2 n} \tau$ ( $n=$ stress exponent) [6]. The straight line refers to the relation $d=K / \sigma$, with $K=800 \mu \mathrm{m}$ MPa. Compression data are from [29].

in room temperature compression the grain size is around $0.8 \mu \mathrm{m}$. Even smaller grain structures should be expected in high pressure torsion of $\mathrm{NiAl}$ at room temperature. Moreover, a high dislocation density and/or non-equilibrium grain boundaries may enhance ductility [28].

b) Condition (i) is strictly valid only for a random orientation distribution. Strong textures with soft preferred orientation distributions, as is the case for simple shear deformation of $\mathrm{NiAl}$, should alter this condition. However, grain refinement and texture strengthening work in opposite directions (compare Figs. 3 and $8 \mathrm{~b}$ ).

To check the arguments mentioned above, specific investigations are planned with polycrystalline $\mathrm{NiAl}$ with grain sizes smaller than about $2.5 \mu \mathrm{m}$.

\section{Conclusions}

1. During high-strain torsion of $\mathrm{NiAl}$ at elevated temperatures continuous dynamic recrystallisation leads to grain refinement into the micron range. The steady state fraction of high angle grain boundaries is between $60-$ $80 \%$. At the lowest temperature used the "grain size" is only a factor of 1.2 larger than the subgrain size.

2. The shear texture is characterised by two components, $\{100\}<100>$ (cube) and $\{110\}<100>$ (Goss) increasing with strain to an almost constant strength. The intensity of Goss increases with temperature, while that of the cube decreases independent of the initial orientation.

3. Ductilisation of NiAl seems to be an inherent problem due to a high plastic anisotropy. However, as discussed, under certain conditions grain refinement may help to ductilise the material. This has to be proven by further experiments.

The help of T. Reiter and A. Jurk in sample preparation is gratefully acknowledged. Thanks are due to the Deutsche Forschungsgemeinschaft for funding (Sk21/19-1, 2) and DESY-HASYLAB for beamtime and financial support of travel and accommodation. 


\section{References}

[1] R.D. Noebe, R.R. Bowman, M.V. Nathal, in: N.S. Stoloff, V.K. Sikka (Eds.), Physical Metallurgy and Processing of Intermetallic Compounds, Chapman \& Hall, New York (1996) 212.

[2] E.M. Schulson, D.R. Barker: Scripta Metall. 17 (1983) 519.

[3] R.Z. Valiev, Y. Estrin, Z. Horita, T.G. Langdon, M.J. Zehetbauer, Y.T. Zhu: JOM 58 (2006) 33.

[4] W. Skrotzki, B. Klöden, R. Tamm, C.-G. Oertel, L. Wcislak, E. Rybacki, in: M.J. Zehetbauer, R.-Z. Valiev (Eds.), Nanomaterials by Severe Plastic Deformation, Wiley-VCH, Weinheim (2004) 303.

[5] W. Skrotzki, B. Klöden, R. Tamm, C.-G. Oertel, U. Garbe, E. Rybacki: Textures Microstruct. 35 (2003) 163.

[6] M.S. Paterson, D.L. Olgaard: J. Struct. Geol. 22 (2000) 1341

[7] E. Rybacki, M.S. Paterson, R. Wirth, G. Dresen: J. Geophys. Res. 108 (2003) 2089

[8] H.W. Swift: Engineering 163 (1947) 253.

[9] R. Tamm, M. Lemke, C.-G. Oertel, W. Skrotzki: Mater. Sci. Forum 273-275 (1998) 411

[10] M. Dahms, T. Eschner: Quantitative Texturanalyse durch iterative Reihenzerlegung von Beugungs-Polfiguren (software manual) (1996).

[11] H.-J. Bunge: Texture Analysis in Materials Science, Mathematical Methods, Culliver Verlag, Göttingen (1993).

[12] L.S. Tóth, A. Molinari: Acta Metall. Mater. 42 (1994) 2459.

[13] B. Klöden, E. Rybacki, C.-G. Oertel, W. Skrotzki: Scripta Mater. 52 (2005) 289.

[14] J.G. Ramsay, M.I. Huber: The Techniques of Modern Structural Geology, Academic Press, London (1983).

[15] S. Gourdet, F. Montheillet: Mater. Sci. Eng. A 283 (2000) 274

[16] F. Montheillet, J. Le Coze: Phys. Stat. Sol. (a) 189 (2002) 51.

[17] S. Gourdet, F. Montheillet: Acta Mater. 50 (2002) 2801.

[18] S. Gourdet, F. Montheillet: Acta Mater. 51 (2003) 2685

[19] B. Klöden, W. Skrotzki, C.-G. Oertel, E. Rybacki: Mater. Sci. Forum 495-497 (2005) 743.

[20] W. Skrotzki, P. Haasen, in: R.E. Tressler, R.C. Bradt (Eds.), Deformation of Ceramics II, Plenum Publishing Corporation, New York (1984) 429.
[21] R. von Mises: Z. angew. Math. Mech. 8 (1928) 161.

[22] M.J. Mills, D.B. Miracle: Acta metall. Mater. 41 (1993) 85.

[23] Y.Q. Sun: Phil. Mag. 80 (2000) 447.

[24] D. Caillard, C. Vailhé, D. Farkas: Phil. Mag. 79 (1999) 723.

[25] Y.Q. Sun, N. Yang: Acta Mater. 51 (2003) 5601.

[26] R.D. Noebe, R.R. Bowman, M.V. Nathal: NASA Technical Memorandum 105598 (1992)

[27] W. Skrotzki, R. Tamm, C.-G. Oertel, B. Beckers, H.-G. Brokmeier, E. Rybacki: Mater. Sci. Eng. A 319 (2001) 364.

[28] A.A. Nazarov, A.E. Romanov, R.Z. Valiev: Acta metall. Mater. 41 (1993) 1033.

[29] J. Fischer-Bühner: PhD Thesis, RWTH Aachen, Shaker Verlag, Aachen (1998).

(Received October 2, 2006; accepted February 6, 2007)

Correspondence address

Prof. Dr. Werner Skrotzki

Institut für Strukturphysik

Technische Universität Dresden, D-01062 Dresden, Germany

Tel.: +4935146335144

Fax: +4935146337048

E-mail: werner.skrotzki@physik.tu-dresden.de

You will find the article and additional material by entering the document number MK101471 on our website at www.ijmr.de 